\title{
Corrigendum: Assessing cultural intelligence, personality and identity amongst young white Afrikaans-speaking students: A preliminary study
}

\begin{tabular}{|c|c|}
\hline \multicolumn{2}{|c|}{$\begin{array}{l}\text { Authors: } \\
\text { Natasha } \mathrm{Nel}^{1} \\
\text { J. Alewyn } \mathrm{Nel}^{1} \\
\text { Byron G. Adams } \\
\text { Leon T. de Beer }\end{array}$} \\
\hline $\begin{array}{l}\text { Affiliations: } \\
{ }^{1} \text { WorkWell Re } \\
\text { North-West U } \\
\text { Potchefstroom } \\
\text { South Africa }\end{array}$ & $\begin{array}{l}\text { search Unit, } \\
\text { niversity, } \\
\text { Campus, }\end{array}$ \\
\hline \multicolumn{2}{|c|}{$\begin{array}{l}{ }^{2} \text { Department of Culture } \\
\text { Studies, Tilburg University, } \\
\text { the Netherlands }\end{array}$} \\
\hline \multicolumn{2}{|c|}{$\begin{array}{l}{ }^{3} \text { Department of Industrial } \\
\text { Psychology and People } \\
\text { Management, University of } \\
\text { Johannesburg, Kingsway } \\
\text { Campus, South Africa }\end{array}$} \\
\hline \multicolumn{2}{|c|}{$\begin{array}{l}\text { Corresponding author: } \\
\text { Leon de Beer, } \\
\text { debeer.leon@nwu.ac.za }\end{array}$} \\
\hline \multicolumn{2}{|c|}{$\begin{array}{l}\text { Dates: } \\
\text { Published: } 30 \text { Nov. } 2017\end{array}$} \\
\hline \multicolumn{2}{|c|}{$\begin{array}{l}\text { How to cite this article: } \\
\text { Nel, N., Nel, J.A., Adams, } \\
\text { B.G., \& De Beer, L.T. (2017). } \\
\text { Corrigendum: Assessing } \\
\text { cultural intelligence, } \\
\text { personality and identity } \\
\text { amongst young white } \\
\text { Afrikaans-speaking students: } \\
\text { A preliminary study. SA } \\
\text { Journal of Human Resource } \\
\text { Management/SA Tydskrif vir } \\
\text { Menslikehulpbronbestuur, } \\
\text { 15(0), a1008. https://doi. } \\
\text { org/10.4102/sajhrm. } \\
\text { v15i0.1008 }\end{array}$} \\
\hline \multicolumn{2}{|l|}{ Read online: } \\
\hline 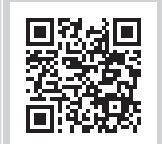 & $\begin{array}{l}\text { Scan this QR } \\
\text { code with your } \\
\text { smart phone or } \\
\text { mobile device } \\
\text { to read online. }\end{array}$ \\
\hline
\end{tabular}

In the version of this article initially published, the correlation in Table 2 between '1. Cognitive $\mathrm{CQ}^{\prime}$ (row 1, column 1) and '5. Conscientiousness' (row 5, column 1) was mistakenly reported as 1.13. The actual value should have been 0.13 . Similarly, the correlation between ' 3 . Motivational CQ' (row 3, column 3) and '7. Extroversion' (row 7, column 3) was mistakenly reported as 1.21. The actual value should have been 0.21. Lastly, the correlation between '2. Metacognitive CQ' (row 2, column 2) and '6. Emotional stability' (row 6, column 2) was mistakenly reported as 1.30. The actual value should have been 0.30. An updated Table 2 is presented here:

TABLE 2: Correlation coefficients between cultural intelligence, personality and identity.

\begin{tabular}{lllllllllllllllll}
\hline Variable & $\mathbf{1}$ & $\mathbf{2}$ & $\mathbf{3}$ & $\mathbf{4}$ & $\mathbf{5}$ & $\mathbf{6}$ & $\mathbf{7}$ & $\mathbf{8}$ & $\mathbf{9}$ & $\mathbf{1 0}$ & $\mathbf{1 1}$ & $\mathbf{1 2}$ & $\mathbf{1 3}$ & $\mathbf{1 4}$ & $\mathbf{1 5}$ \\
\hline 1. Cognitive $\mathrm{CQ}$ & 1.00 & - & - & - & - & - & - & - & - & - & - & - & - & - & - \\
2. Metacognitive $\mathrm{CQ}$ & $0.42 \dagger$ & 1.00 & - & - & - & - & - & - & - & - & - & - & - & - & - \\
3. Motivational $\mathrm{CQ}$ & $0.48 \dagger$ & $0.54 \dagger$ & 1.00 & - & - & - & - & - & - & - & - & - & - & - & - \\
4. Behavioural $\mathrm{CQ}$ & $0.52 \dagger$ & $0.55 \dagger$ & $0.58 \dagger$ & 1.00 & - & - & - & - & - & - & - & - & - & - & - \\
5. Conscientiousness & 0.13 & $0.33 \dagger$ & 0.25 & $0.31 \dagger$ & 1.00 & - & - & - & - & - & - & - & - & - & - \\
6. Emotional stability & 0.28 & 0.30 & 0.26 & $0.34 \dagger$ & $0.35 \dagger$ & 1.00 & - & - & - & - & - & - & - & - & - \\
7. Extroversion & 0.24 & $0.31 \dagger$ & 0.21 & $0.31 \dagger$ & $0.51 \dagger$ & $0.57 \dagger$ & 1.00 & - & - & - & - & - & - & - & - \\
8. Facilitating & 0.13 & $0.40 \dagger$ & $0.30 \dagger$ & 0.26 & $0.45 \dagger$ & $0.39 \dagger$ & $0.49 \dagger$ & 1.00 & - & - & - & - & - & - & - \\
9. Integrity & 0.15 & 0.22 & 0.16 & 0.20 & $0.38 \dagger$ & $0.47 \dagger$ & $0.57 \dagger$ & $0.38 \dagger$ & 1.00 & - & - & - & - & - & - \\
10. Intellect & 0.18 & $0.47 \dagger$ & $0.34 \dagger$ & $0.33 \dagger$ & $0.66 \dagger$ & $0.40 \dagger$ & $0.60 \dagger$ & $0.61 \dagger$ & $0.40 \dagger$ & 1.00 & - & - & - & - & - \\
11. Openness & 0.19 & $0.42 \dagger$ & $0.31 \dagger$ & $0.32 \dagger$ & $0.67 \dagger$ & $0.40 \dagger$ & $0.63 \dagger$ & $0.64 \dagger$ & $0.46 \dagger$ & $0.82 \dagger$ & 1.00 & - & - & - & - \\
12. Relationship & 0.29 & 0.25 & 0.27 & $0.34 \dagger$ & 0.17 & $0.58 \dagger$ & $0.56 \dagger$ & $0.33 \dagger$ & $0.40 \dagger$ & $0.42 \dagger$ & $0.40 \dagger$ & 1.00 & - & - & - \\
harmony & & & & & & & & & & & & & &
\end{tabular}

$\mathrm{CQ}$, Cultural intelligence.

$\dagger$, Correlation is significant at the 0.01 level.

\$. Correlation is practically significant $r \geq 0.30$ (medium effect).

The error has been corrected in the PDF version of the article. The authors apologise for any inconvenience caused.
Note: Doi of original article: http://dx.doi.org/10.4102/sajhrm.v13i1.643

Copyright: ( 2017. The Authors. Licensee: AOSIS. This work is licensed under the Creative Commons Attribution License. 